\title{
Desenvolvimento do índice de qualidade da água bruta para fins de abastecimento público-IPA para Amazônia
}

\author{
Development of Raw Water Quality Index for Public Supply-IPA for amazon
}

\author{
A. F. S. Oliveira ${ }^{1}$; L. D. P. Silva ${ }^{2}$; T. M. Silva ${ }^{3}$; L. P. Santos ${ }^{4}$; R. M. Rocha ${ }^{5}$ K K.; \\ H. G. Freitas ${ }^{1}$; S. F. P. Pereira ${ }^{2}$ \\ ${ }^{1}$ Faculdade de Química, Universidade Federal do Pará, 66075-110, Belém-Pará, Brasil \\ ${ }^{2}$ Faculdade de Engenharia de Materiais, Universidade Federal do Pará- Campus de Ananideua, 66075-900, \\ Ananideua-Pará, Brasil \\ ${ }^{3}$ Programa de Pós Graduação em Química da Universidade Federal do Pará, 66075-110, Belém-Pará, Brasil \\ ${ }^{4}$ Instituto Socioambiental de Recursos Hídricos, Universidade Federal Rural da Amazônia, 66.077-830, Belém-Pará, \\ Brasil \\ ${ }^{5}$ Secretaria de Saude Publica do Pará, Laboratório Central do Pará, 66.823-010, Belém-Pará, Brasil \\ *heloizy@ufpa.br
}

(Recebido em 28 de novembro de 2016; aceito em 22 de dezembro de 2016)

\begin{abstract}
A Amazônia, cujos dois terços de sua área estão situados no Brasil, é foco de intensas políticas de construção de hidrelétricas com objetivo de satisfazer a demanda energética do país. Todavia, tais políticas implicam em problemas socioambientais para a região, incluindo a perda da biodiversidade, alteração do regime pluviométrico, perda da atividade pesqueira, dentre outros. Nessa perspectiva, a avaliação da qualidade da água das represas que constituem essas hidrelétricas torna-se crucial na avaliação do grau de comprometimento dos ecossistemas aquáticos por serem estes ambientes vitais no equilíbrio ecológico da região amazônica. O objetivo deste trabalho foi elaborar um Índice de Qualidade da Água Bruta para Fins de Abastecimento Público (IPA) específico para a região Amazônica com base em dados de variáveis químicas e físico-químicas de três represas de hidrelétricas situadas na Amazônia: Tucuruí (PA), Curuá-una (PA) e Samuel (RO). Realizaram-se quatro campanhas com base nos períodos chuvoso e estiagem. Os resultados mostraram que as águas dos reservatórios de Tucuruí e Curuá-Una encontram-se em boas condições de abastecimento público após tratamento simplificado, enquanto que a represa de Samuel encontra-se em condição aceitável, o que sugere que apesar dos danos ocasionados durante os primeiros anos de operação dessas represas, ocorre certa melhoria da qualidade dessas águas. Este trabalho contribui como ferramenta de base para outros estudos voltados para a compreensão da dinâmica ambiental em ambientes impactados na Amazônia, bem como oferece uma excelente ferramenta, IPA, para o desenvolvimento de outros estudos em ambientes naturais nesta região.
\end{abstract}

Palavras-chave: Indice de Qualidade de Água, Abastecimento, IPA, Reservatórios.

The Amazon, whose two-thirds of its area are situated in Brazil, is focus of intense hydroelectric construction policies in order to satisfy the energy demand of the country. However, these policies imply environmental problems for the region, including loss of biodiversity, altered rainfall regime, loss of fishing activity, among other. In this perspective, the evaluation of the quality of water from UHE reservoir becomes crucial in assessing the degree of commitment of aquatic ecosystems for these vital environments in the ecological balance of the Amazon region. The objective of this work was to elaborate a Raw Water Quality Index for Public Supply (IPA) to Amazon region based on chemical and physicochemical variables of three reservoir located in the Amazon: Tucuruí (PA ), Curuá-Una (PA) and Samuel (RO). Performed four campaigns based on rainy and rainless periods. The results showed that the waters of the reservoirs of Tucuruí and Curuá-Una are in good condition of public supply after simplified treatment, while the Samuel's reservoir is in acceptable condition, suggesting that despite the damage caused during the first years of operation of these dams, occured some improvement of the quality of these waters. This work contributes as a basis for other studies aimed at understanding the dynamics in the Amazon impacted environments, as well as offers an excellent tool, IPA, to the development of other studies in natural environments in this region.

Keywords: Raw water quality index for public supply, IPA. 


\section{INTRODUÇÃO}

A água é um recurso natural de fundamental importância para a vida no planeta e indispensável para o desenvolvimento da sociedade, no entanto apesar de sua abundância, apenas uma pequena quantidade está disponível para o consumo humano. Segundo a Agência Nacional de Águas - ANA (2006) [1], o planeta não está perdendo água, mas a qualidade disponível está diminuindo, os mananciais utilizados para diversos fins estão sendo comprometidos pelo lançamento de esgotos, originando organismos patogênicos e substâncias tóxicas, tornando essa água inapropriada para o consumo humano. Nesse contexto, monitorar os recursos hídricos é de grande importância para a manutenção da qualidade da água e para o bem estar humano.

Para o monitoramento da qualidade dos corpos hídricos, vários índices são aplicados, representados através de cálculos que utilizam valores dos parâmetros analisados das amostras coletadas do corpo d'água em estudo. De acordo com a Companhia Ambiental do Estado de São Paulo (CETESB) [4], o Índice de qualidade da água bruta para fins de abastecimento público (IPA) é o produto da ponderação dos resultados atuais do Índice de Qualidade de Águas (IQA) e do Índice de Substâncias Tóxicas e Organolépticas(ISTO), que é composto pelo grupo de substâncias que afetam a qualidade organoléptica da água, bem como de substâncias tóxicas. Vale ressaltar que as substâncias tóxicas são aquelas capazes de provocar a morte ou danos à saúde humana se ingeridas, inaladas ou por contato com a pele, mesmo em pequenas quantidades [3]. Já as propriedades organolépticas são aquelas que podem ser percebidas pelos sentidos humanos, por exemplo, olfato e paladar [5].

Em termos de recursos hídricos, o Brasil é privilegiado neste quesito, porém, sua disponibilidade é desigual, pois a região amazônica concentra $81 \%$ desses recursos, mas também tem o menor contingente populacional [6]. Diversas bacias hidrográficas estão localizadas em seu território como as bacias do Paraná, Araguaia-Tocantins, Parnaíba e Amazônica, sendo esta última, a maior do mundo. As propriedades da água de excelente solvente e também sua capacidade de transportar partículas, acabam por integrar a si diversas impurezas, as quais definem sua qualidade. Através do monitoramento ambiental dos recursos hídricos em sistemas lóticos e lênticos e obtenção de seus parâmetros químicos, físico-químicos e biológicos, permitem a partir destes valores, detectar tendências ou alterações na qualidade hídrica [7].

A alteração na qualidade e desequilíbrio dos corpos d'água pode ocorrer de forma natural ou antrópica, como o despejo de resíduos tóxicos nos rios por indústrias ou de efluentes diretamente lançados no mesmo. Muitos corpos d’água foram tratados como receptores residuais e ainda são vistos desta forma, tendo como consequências a poluição, redução da biodiversidade de lagos e rios e agravando o risco à saúde humana [8]. Desta forma, a importância de analisar periodicamente a qualidade dos corpos d'água destas regiões que sofreram ou ainda sofrem alterações expressivas são essenciais, uma vez que, diversas comunidades locais utilizam para suas necessidades diárias.

Diante da problemática apresentada, desenvolveu-se um trabalho a fim de analisar a qualidade das águas em áreas inundadas de Usinas de Hidrelétrica - UHE, o qual foi utilizado neste trabalho o IPA - Índice de Qualidade da água bruta para fins de abastecimento.

\section{MATERIAL E MÉTODOS}

As áreas de estudo foram os reservatórios das UHEs Tucuruí, Curuá-Una, ambas no Pará e Samuel, em Rondônia. Para a realização das coletas ao longo das represas utilizaram-se pontos georreferenciados através de um sistema de posição global GPS (Global Positionning System). As coletas foram divididas em quatro campanhas em intervalo trimestral, obedecendo ao período de maior e menor precipitação pluviométrica durante o ano de 2015 e 2016 sendo 16 estações de coleta em Tucuruí, 8 em Curuá-Una e 11 em Samuel, totalizando 35 estações.

As amostras de água foram coletadas através de garrafa modelo "Van Dorn" (5 litros), obedecendo a critérios preconizados pela CETESB (1988) [2]. Algumas variáveis foram analisadas in situ, como OD, $\mathrm{pH}$, temperatura e turbidez, enquanto que as demais foram 
analisadas em laboratório. As amostras foram coletadas em garrafas de polietileno com capacidade de 1 litro, previamente descontaminadas com solução de ácido nítrico, lavadas com água destilada e secas, etiquetadas e ambientadas com água da coleta. Após a coleta, as amostras foram acondicionadas sob refrigeração e encaminhadas para o Laboratório de Química Analítica e Ambiental (LAQUANAM-UFPA) para tratamento e análise.

-Localização

A UHE de Tucuruí (Figura 1) situa-se na bacia hidrográfica do Tocantins-Araguaia localizado quase que integralmente entre os paralelos $2^{\circ}$ e $18^{\circ}$ e os meridianos de longitude oeste $46^{\circ}$ e $56^{\circ}$.

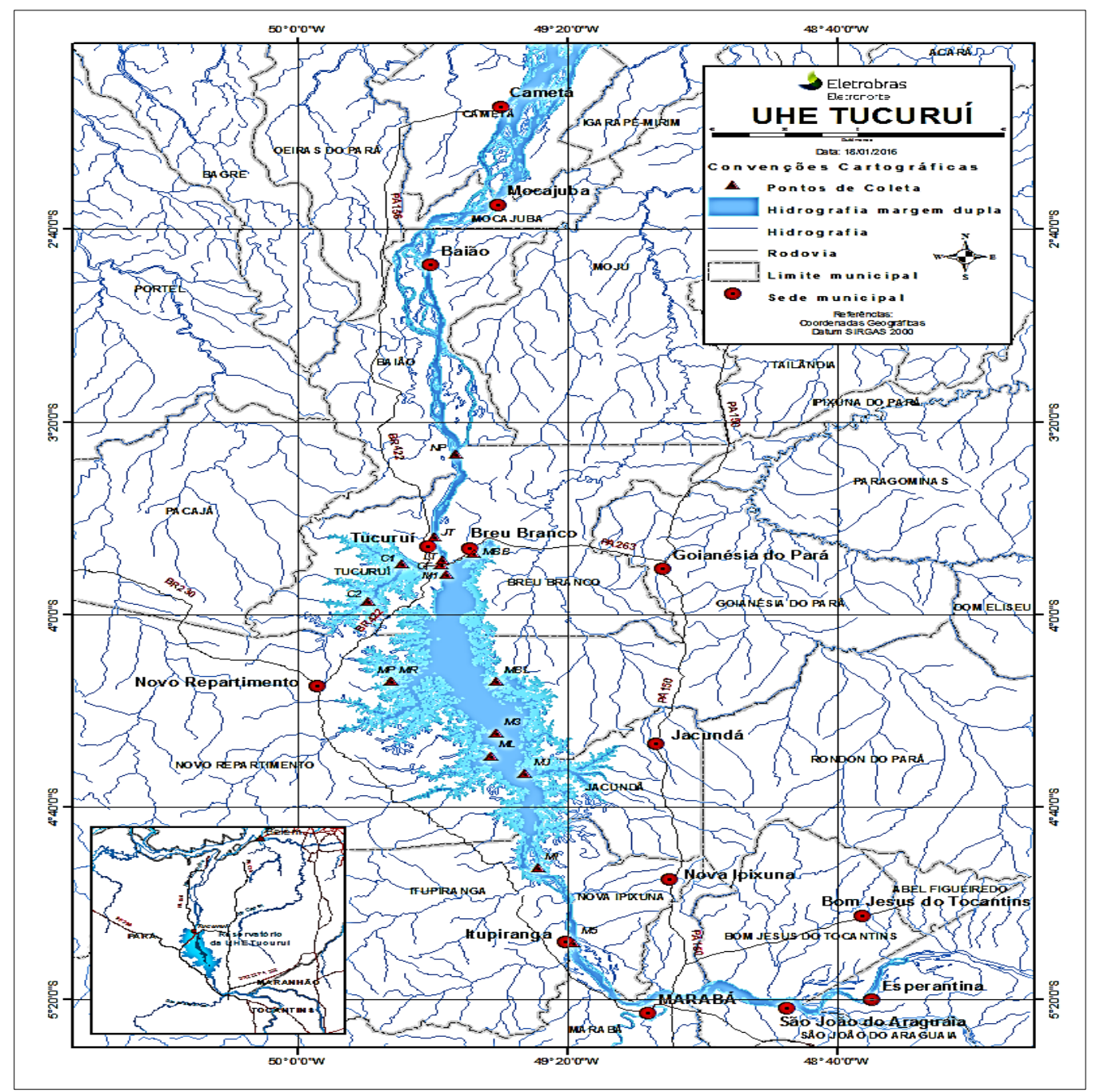

Figura 1 - Mapa de localização do reservatório de Tucuruí - PA

Fonte: ELETRONORTE, 2016

A identificação das estações de coleta, bem como a localização geográfica de cada uma delas estão apresentadas na Tabela 1. 
Tabela 1: Identificação e Localização das Estações de Coleta - Tucuruí

\begin{tabular}{|c|c|c|c|}
\hline IDENTIFICAÇÃO & ESTAÇÃO DE COLETA & LATITUDE (S) & LONGITUDE (W) \\
\hline $\mathrm{C} 1$ & Caraipé 1 & $03^{\circ} 49^{\prime} 26,51^{\prime \prime}$ & $49^{\circ} 44^{\prime} 38,51^{\prime \prime}$ \\
\hline $\mathrm{C} 2$ & Caraipé 2 & $03^{\circ} 57^{\prime} 12,1^{\prime \prime}$ & $49^{\circ} 49^{\prime} 31,1^{\prime \prime}$ \\
\hline M1 & Montante 1 & $03^{\circ} 51^{\prime} 38,8^{\prime \prime}$ & $49^{\circ} 37^{\prime} 56,6^{\prime \prime}$ \\
\hline M3 & Montante 3 & $04^{\circ} 24^{\prime} 38,5^{\prime \prime}$ & $49^{\circ} 30^{\prime} 37,8^{\prime \prime}$ \\
\hline M5 & Montante 5 & $05^{\circ} 08^{\prime} 07,2^{\prime \prime}$ & $49^{\circ} 19^{\prime} 19,5^{\prime \prime}$ \\
\hline MBL & Montante Belauto & $04^{\circ} 13^{\prime} 57,2^{\prime \prime}$ & $49^{\circ} 30^{\prime} 37,8^{\prime \prime}$ \\
\hline MBB & Montante Breu Branco & $03^{\circ} 47^{\prime} 17,6^{\prime \prime}$ & $49^{\circ} 34^{\prime} 01,4^{\prime \prime}$ \\
\hline MP & Montante Pucuruí (C) & $04^{\circ} 13 ' 57,2^{\prime \prime}$ & $49^{\circ} 46^{\prime} 08,6^{\prime \prime}$ \\
\hline MR & Montante Repartimento & $04^{\circ} 13^{\prime} 16,3^{\prime \prime}$ & $49^{\circ} 41^{\prime} 59,6^{\prime \prime}$ \\
\hline MI & Montante Ipixuna & $04^{\circ} 52^{\prime} 35,2^{\prime \prime}$ & $49^{\circ} 24^{\prime} 31,2^{\prime \prime}$ \\
\hline MJ & Montante Jacundá Velho & $04^{\circ} 33^{\prime} 00,5^{\prime \prime}$ & $49^{\circ} 26^{\prime} 20,6^{\prime \prime}$ \\
\hline ML & Montante Lontra & $04^{\circ} 29^{\prime} 23,9^{\prime \prime}$ & $49^{\circ} 31^{\prime} 27,7^{\prime \prime}$ \\
\hline $\mathrm{CF}$ & Canal de Fuga & $03^{\circ} 49^{\prime} 45,5^{\prime \prime}$ & $49^{\circ} 38^{\prime} 53,7^{\prime \prime}$ \\
\hline $\mathrm{LT}$ & Linha de Transmissão & $03^{\circ} 48^{\prime} 42,9^{\prime \prime}$ & $49^{\circ} 38^{\prime} 32^{\prime \prime}$ \\
\hline JT & Jusante Tucuruí & $03^{\circ} 43^{\prime} 57,7^{\prime \prime}$ & $49^{\circ} 39^{\prime} 45,2^{\prime \prime}$ \\
\hline NP & Nazaré dos Patos (C) & $03^{\circ} 26^{\prime} 47,4^{\prime \prime}$ & $49^{\circ} 36^{\prime} 31,4^{\prime \prime}$ \\
\hline
\end{tabular}

A UHE Curuá-Una (Figura 2) localiza-se a cerca de $850 \mathrm{~km}$, em linha reta a oeste, da capital do Estado do Pará. Suas coordenadas geográficas são: 2²4'52" S e 54²42'36" W.

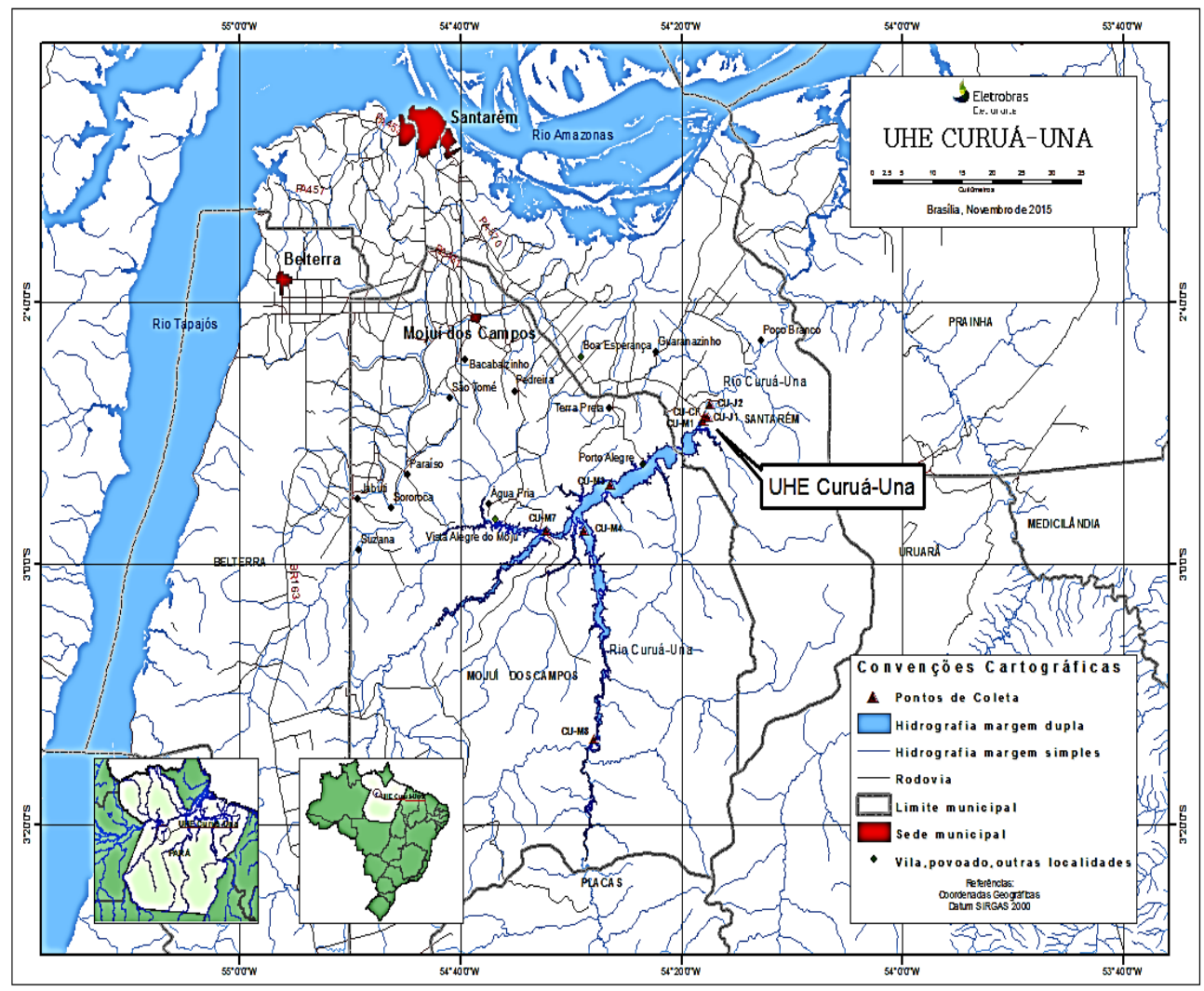

Figura 2- Mapa de localização do reservatório de Curuá-Una - Santarém - PA Fonte: ELETRONORTE, 2016

A localização e a identificação das estações de coleta no reservatório de Curuá-Una estão apresentadas na Tabela 2. 
Tabela 2: Identificação e Localização das Estações de Coleta - Curuá-Una

\begin{tabular}{cccc}
\hline IDENTIFICAÇÃO & ESTAÇÃO DE COLETA & LATITUDE (S) & LONGITUDE (W) \\
\hline CU-M1 & Montante 1 & $02^{\circ} 49^{\prime} 02,6^{\prime \prime}$ & $54^{\circ} 18^{\prime} 02,6^{\prime \prime}$ \\
CU-M3 & Montante 3 & $02^{\circ} 54^{\prime} 00,0^{\prime \prime}$ & $54^{\circ} 26^{\prime} 24,0^{\prime \prime}$ \\
CU-M4 & Montante 4 & $02^{\circ} 57^{\prime} 29,5^{\prime \prime}$ & $54^{\circ} 28^{\prime} 48,6^{\prime \prime}$ \\
CU-M7 & Montante 7 & $02^{\circ} 57^{\prime} 33,3^{\prime \prime}$ & $54^{\circ} 32^{\prime} 12,0^{\prime \prime}$ \\
CU-M8 & Montante 8 & $03^{\circ} 13^{\prime} 28,9^{\prime \prime}$ & $54^{\circ} 27^{\prime} 53,5^{\prime \prime}$ \\
CU-CF & Canal de Fuga & $02^{\circ} 48^{\prime} 42,0^{\prime \prime}$ & $54^{\circ} 17^{\prime} 53,8^{\prime \prime}$ \\
CU-J1 & Jusante 1-Rio Curuá-Una & $02^{\circ} 48^{\prime} 45,2^{\prime \prime}$ & $54^{\circ} 17^{\prime} 40,2^{\prime \prime}$ \\
CU-J2 & Jusante 2-Rio Curuá-Una & $02^{\circ} 47^{\prime} 50,6^{\prime \prime}$ & $54^{\circ} 17^{\prime} 24,2^{\prime \prime}$ \\
\hline
\end{tabular}

A barragem de Samuel (Figura 3) está situada no Rio Jamari na Cachoeira de Samuel (8o 45' S 63o 25' W), 52 km da cidade de Porto Velho, Rondônia.

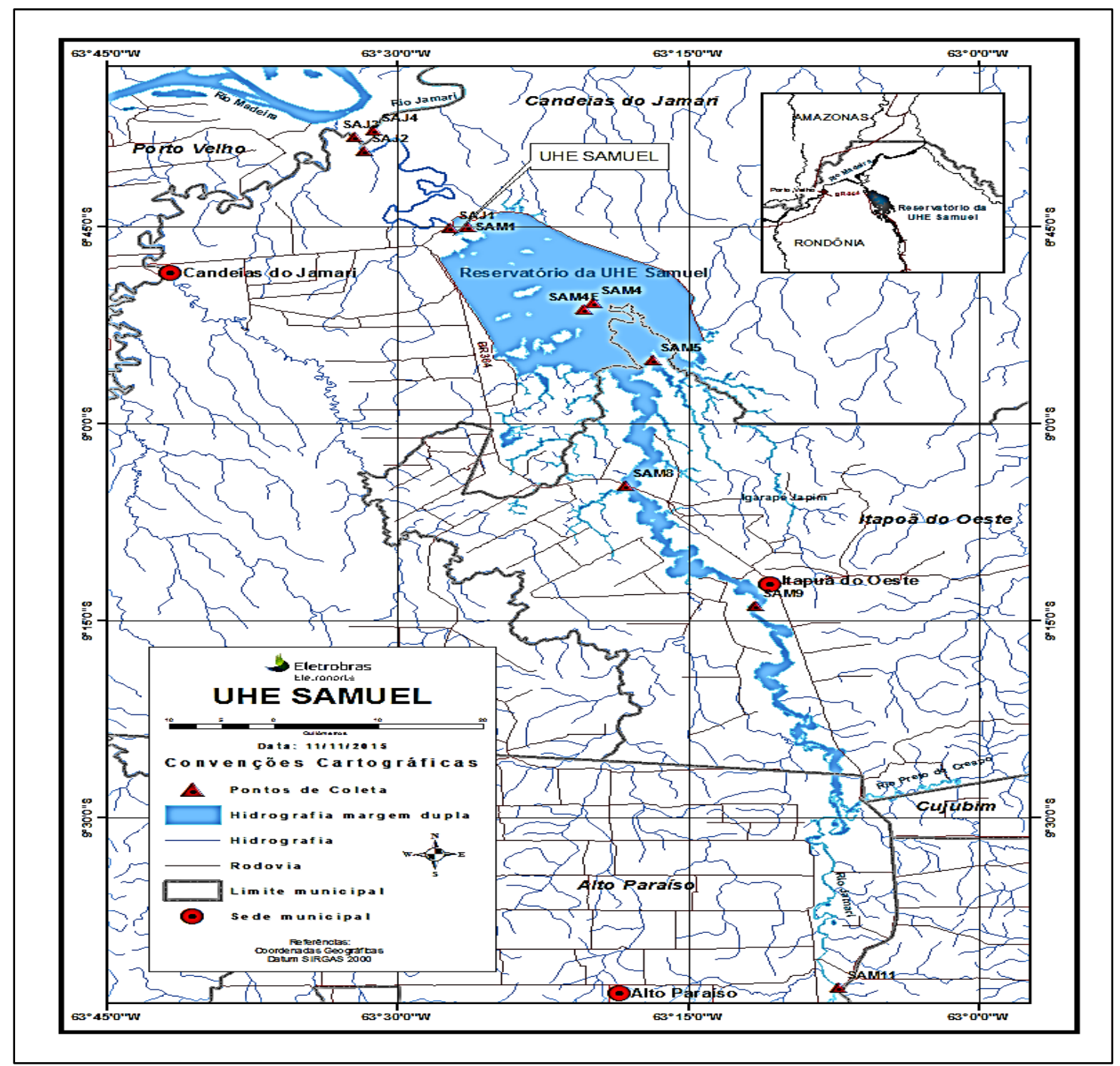

Figura 3-: Mapa de localização do reservatório de Samuel - Porto Velho - RO Fonte: ELETRONORTE, 2016

As estações de coleta no reservatório de Samuel estão identificadas na Tabela 3. 
Tabela 3: Identificação e Localização das Estações de Coleta - Samuel

\begin{tabular}{cccc}
\hline IDENTIFICAÇÃOO & NOME DO SÍTIO & LATITUDE (S) & LONGITUDE (W) \\
\hline SA-M1 & Montante 1 & $08^{\circ} 45^{\prime} 02,0^{\prime \prime}$ & $63^{\circ} 26^{\prime} 26,5^{\prime \prime}$ \\
SA-M4 & Montante 4 & $08^{\circ} 50^{\prime} 48,8^{\prime \prime}$ & $63^{\circ} 19^{\prime} 56,8^{\prime \prime}$ \\
SA-M4E & Montante 4 margem esquerda & $08^{\circ} 51^{\prime} 17,9^{\prime \prime}$ & $63^{\circ} 20^{\prime} 25,6^{\prime \prime}$ \\
SA-M5 & Montante 5 & $08^{\circ} 55^{\prime} 09,2^{\prime \prime}$ & $63^{\circ} 16^{\prime} 51,8^{\prime \prime}$ \\
SA-M8 & Montante 8 & $09^{\circ} 04^{\prime} 46,4^{\prime \prime}$ & $63^{\circ} 18^{\prime} 15,7^{\prime \prime}$ \\
SA-M9 & Montante 9 & $09^{\circ} 13^{\prime} 51,5^{\prime \prime}$ & $63^{\circ} 11^{\prime} 34,9^{\prime \prime}$ \\
SA-M11 & Montante 11 & $9^{\circ} 42^{\prime} 57.92^{\prime \prime}$ & $63^{\circ} 7^{\prime} 18.12^{\prime \prime}$ \\
SA-J1 & Jusante 1 - Rio Jamari & $08^{\circ} 45^{\prime} 04,4^{\prime \prime}$ & $63^{\circ} 27^{\prime} 20,0^{\prime \prime}$ \\
SA-J2 & Jusante 2 - Rio Jamari & $08^{\circ} 39^{\prime} 14,6^{\prime \prime}$ & $63^{\circ} 31^{\prime} 46,2^{\prime \prime}$ \\
SA-J3 & Jusante 3 - Rio Candeias & $08^{\circ} 38^{\prime} 06,8^{\prime \prime}$ & $63^{\circ} 32^{\prime} 14,7^{\prime \prime}$ \\
SA-J4 & Jusante 4 - Rio Jamari & $08^{\circ} 37^{\prime} 39,6^{\prime \prime}$ & $63^{\circ} 31^{\prime} 17,7^{\prime \prime}$ \\
\hline
\end{tabular}

\section{- Cálculo do IPA}

O IPA foi calculado para os pontos de amostragem dos rios e reservatórios que são utilizados para o abastecimento público. O IPA é o produto da ponderação do IQA (Índice de Qualidade de Águas) e do ISTO (Índice de Substâncias Tóxicas e Organolépticas), de acordo com a Equação 1.

$$
I P A=I Q A x I S T O \quad \text { (Equação 1) }
$$

Nesse contexto, o Índice será composto por três grupos de variáveis: IQA (Temperatura da Água, pH, Oxigênio Dissolvido, Demanda Bioquímica de Oxigênio, Coliformes, Termotolerantes, Nitrogênio Total, Fósforo Total, Resíduo Total e Turbidez), ISTO, que envolve variáveis que indicam a presença de substâncias tóxicas (Cádmio, Chumbo, Cromo Total, Bário, Mercúrio e Níquel) e variáveis que afetam a qualidade organoléptica (Ferro Dissolvido, Manganês, Alumínio Dissolvido, Cobre Dissolvido e Zinco).

O cálculo do ISTO é representado pela Equação 2:

Onde:

$$
I S T O=S T x S O \quad \text { (Equação } 2)
$$

ST - É a ponderação do grupo de substâncias tóxicas (ST) obtida através da multiplicação dos dois valores mínimos mais críticos do grupo de variáveis que indicam a presença dessas substâncias na água (Equação 3):

ST = Mín - 1 (qTA; qTHMFP; qCd; qCr; qPb; qNi; qHg; qNCC, ) x Mín 2(qTA; qTHMFP; qCd; qCr; qPb; qNi; qHg; qNCC) (Equação 3)

SO - É a ponderação do grupo de substâncias organolépticas (SO) obtida através da média aritmética das qualidades padronizadas das variáveis pertencentes a este grupo (Equação 4).

$$
\mathrm{SO}=\overline{\mathrm{X}}(\mathrm{qAl} ; \mathrm{qCu} ; \mathrm{qZn} ; \mathrm{qFe} ; \mathrm{qMn}) \quad \text { (Equação } 4)
$$

O valor do IPA pode variar de 0 a 100, levando em conta que quanto maior for o índice, melhor se apresenta a qualidade da água, como apresentado na Tabela 4. 
Tabela 4: Categoria de Qualidade para IPA

\begin{tabular}{cc}
\hline Categoria & Ponderação \\
\hline Ótima & $79<\mathrm{IPA} \leq 100$ \\
Boa & $51<\mathrm{IPA} \leq 79$ \\
Aceitável/Regular & $36<\mathrm{IPA} \leq 51$ \\
Ruim & $19<\mathrm{IPA} \leq 36$ \\
Péssima & $\mathrm{IPA}<19$ \\
\hline
\end{tabular}

\section{RESULTADOS E DISCUSSÃO}

Na Tabela 5 são apresentados os valores calculados para IPA e sua classificação segundo a CETESB (2010) para os reservatórios de Tucuruí, Curuá-una e Samuel. De acordo com esses dados, o IPA anual para o reservatório de Tucuruí e Curuá-Una, foram classificados como BOM para o abastecimento público. Porém, o reservatório de Samuel foi classificado como ACEITÁVEL/REGULAR para após o tratamento convencional.

Tabela 5: IPA Calculado e sua Classificação

\begin{tabular}{ccccc}
\hline & IQA & ISTO & IPA & Classificação \\
\hline UHE-Tucuruí & 57,18 & 0,986 & 56,37 & Boa \\
UHE-Curuá-una & 52,53 & 0,994 & 52,2 & Boa \\
UHE-Samuel & 53,16 & 0,929 & 49,42 & Aceitável/Regular \\
\hline
\end{tabular}

Nas Figuras 4, 5 e 6 estão apresentadas as médias dos IPAs para cada estação de coleta em cada reservatório. As linhas vermelhas indicam o limite superior BOM, sendo que todos os pontos que estiverem acima dessa linha são considerados de qualidade BOA, e os que tiverem abaixo estão em qualidade ACEITÁVEL/REGULAR.

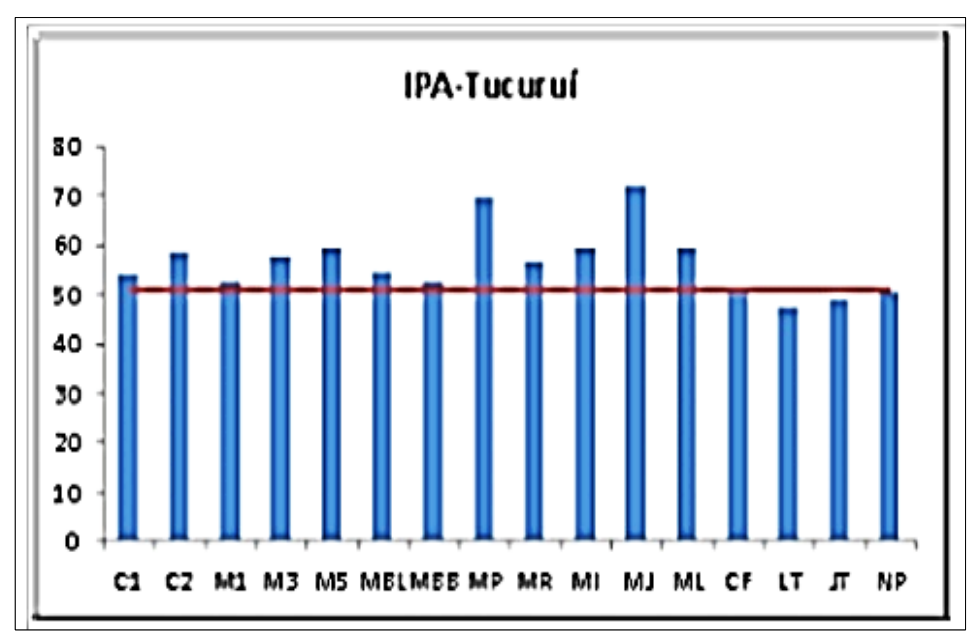

Figura 4-IPA UHE Tucuruí 


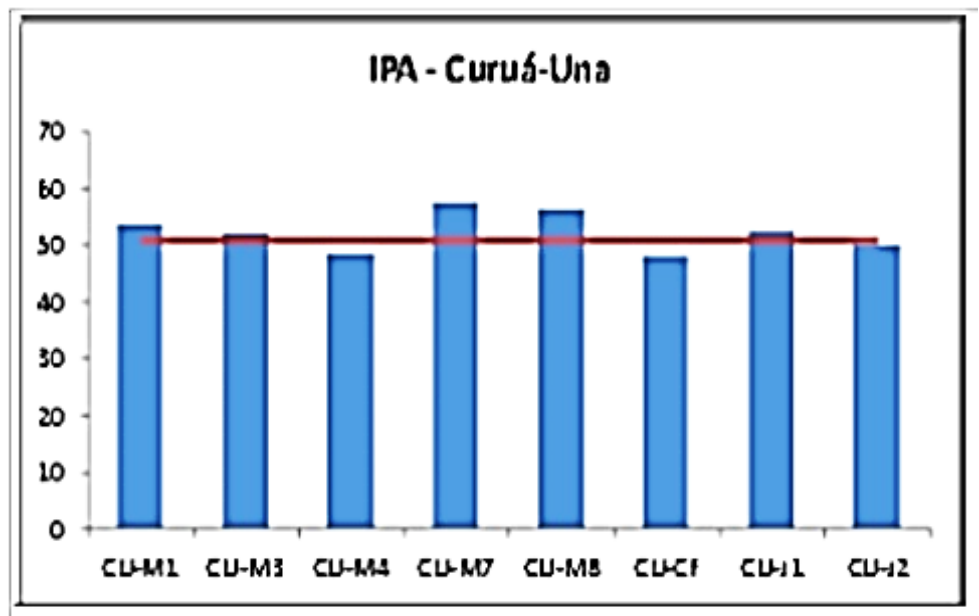

Figura 5-IPA UHE Curuá-una

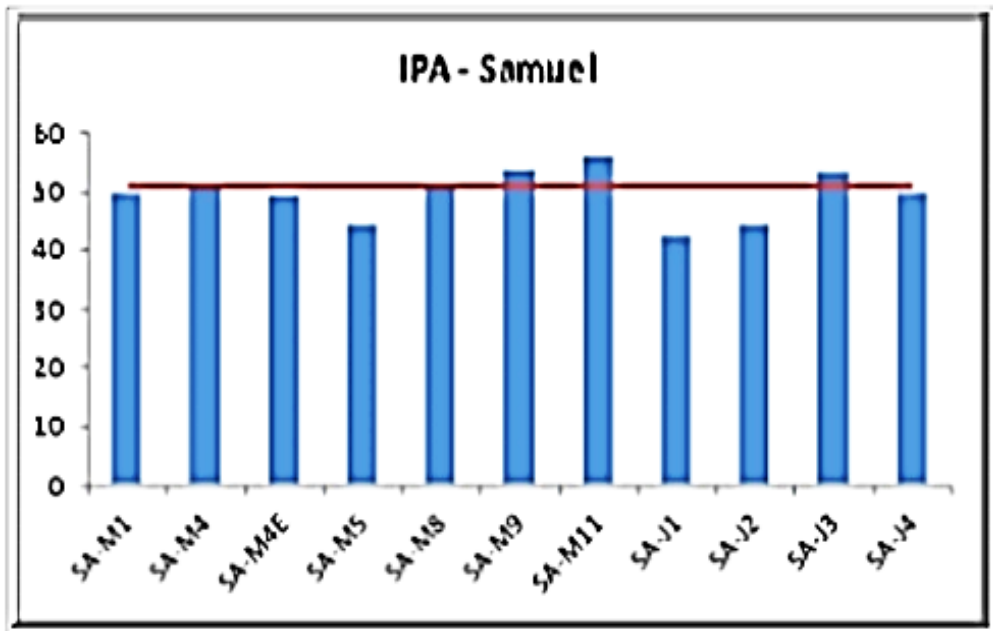

Figura 6-IPA Samuel

Observa-se que na Figura 4 a grande maioria das estações de coletas no reservatório de Tucuruí foi classificada como BOM para o abastecimento público após tratamento convencional com exceção dos pontos CF - Canal de Fuga, LT- Linha de Transmissão, JT- Jusante Tucuruí e NP - Nazaré dos Patos. Os pontos CF e NP obtiveram índices com valores de 50,02 e 50,53, respectivamente. Esses resultados indicaram que a água está margeando o limite entre o nível BOM e o ACEITÁVEL/REGULAR.

Os pontos LT e JT foram classificados como ACEITÁVEL/REGULAR com valores de IPAs de 47,12 e 48,76, respectivamente. O ponto LT é situado na calha do Rio Tocantins, próximo a cidade de Tucuruí, onde neste ponto há lançamento de esgoto, porém como o volume de água é alto possivelmente ocorre o efeito de diluição desses efluentes de esgoto. Já o ponto de coleta JT encontra-se afastada das margens que possuem vegetação alta, apresentando expressiva turbulência com características de ambiente lótico.

Para o IPA de Curuá-Una apresentado na Figura 5, os pontos Cu-M4, CU-CF e CU-J2 obtiveram valores médios de IPAs iguais a 48,41; 47,85 e 49,87 classificados como ACEITÁVEL/REGULAR. Todos com valores próximos ao limite inferior de classificação BOM para IPA.

A Figura 6 mostra que a classificação dos pontos de coletas quanto à qualidade para o abastecimento publico após tratamento convencional alternou ente BOM $\mathrm{e}$ ACEITÁVEL/REGULAR para o reservatório da UHE Samuel. Dentre os pontos classificados como ACEITÁVEL/REGULAR, o ponto SA-J1 (Jusante 1- rio Jamari) foi o que obteve menor valor de IPA 42,42. 


\section{CONCLUSÃO}

Os resultados observados nas amostras de água das UHEs de Tucuruí, Curuá-Una e Samuel, de forma geral, todos os parâmetros estiveram de acordo com os valores estabelecidos pela resolução 357/2005 do CONAMA, podendo-se considerar que a água desses reservatórios são de classe 2.

O IPA calculado permitiu classificar as águas dos reservatórios como BOM e ACEITÁVEL/REGULAR para as UHEs Tucuruí, Curuá-Una e Samuel, respectivamente, o que abre possibilidades de futuros empreendimentos voltados para tratamento e distribuição das águas desses reservatórios à população local.

Este trabalho contribui como ferramenta de base para outros estudos voltados para a compreensão da dinâmica ambiental em ambientes impactados na Amazônia, bem como oferece um excelente método, que é o IPA, para o desenvolvimento de outros estudos em ambientes naturais nesta região.

\section{AGRADECIMENTOS}

\section{À parceria do LAQUANAN/UFPA, LACEN/ELETRONORTE e PIBIC/UFPA.}

\section{REFERÊNCIAS BIBLIOGRÁFICAS}

1. ANA - AGÊNCIA NACIONAL DAS ÁGUAS. Panorama da Qualidade das Águas Superficiais no Brasil, Caderno de Recursos Hídricos, Brasília, SPR. 2006.

2. CETESB - COMPANHIA DE TECNOLOGIA DE SANEAMENTO AMBIENTAL. Amostragem e monitoramento das águas subterrâneas - Norma CETESB, 1988.

3. COMPANHIA DE TECNOLOGIA DE SANEAMENTO AMBIENTAL (CETESB). Qualidade da água: Rios > Índices de Qualidade das Águas > Índices. Disponível em: <http://www.cetesb.sp.gov.br /Agua / rios/indice.asp>. Acesso em: 8 out. 2016.

4. COMPANHIA DE TECNOLOGIA DE SANEAMENTO AMBIENTAL (CETESB). Qualidade da água: Rios > Índices de Qualidade das Águas > Índices. Disponível em: <http://www.cetesb.sp.gov.br /Agua / rios/indice.asp>. Acesso em: 20 mar. 2011.

5. CREMASCO CP, GABRIEL JEF, GABRIEL LRA, FILHO LRAG, GABRIEL LRA, ROMANINI PA. Determinação das funções de pertinência dos índices de qualidade da água e de substâncias tóxicas e organolépticas. Presidente Prudente: Colloquium Exactarum, 2009; Jul/Dez:1(1):46-55, doi: 10.5747/ce.2009.v01.n1.e005.

6. NETO MR, BEZERRA HM, CAMPOS VB, SIQUEIRA K.F, ALMEIDA WL. Avaliação do sistema de tratamento e da qualidade das águas de abastecimento público em Laranjal do Jari, AP. Sci Plena. 2013; 9(11):114301-1-09, doi: http://dx.doi.org/10.14808/sci.plena.2015.081312.

7. ROCHA HM, CABRAL JBP, BRAGA CC. Avaliação Espaço-Temporal das Águas dos Afluentes do Reservatório da UHE Barra dos Coqueiros/Goiás. RBRH - Rev Bras Rec Hídric. 2014; 19(1):131142 .

8. SOUSA, M. F. Qualidade da Água do Canal São Gonçalo-RS/Brasil - Uma Avaliação Hidroquímica Considerando seus Usos Múltiplos [Dissertação]. Pelotas (RS): Universidade Federal de Pelotas; 2015. 105p. 